\title{
ESTUDO DE CASO: Avaliação da importância dos indicadores de desempenho, da comunicação e das ferramentas da qualidade em uma empresa biotecnológica
}

\author{
André Macedo de Almeida Pigosso LUIZ ${ }^{1}$ \\ Eduardo Gomes SALGADO
}

Suzana Eda HIKICHI ${ }^{3}$

\begin{abstract}
${ }^{1}$ Bacharel em Biotecnologia - Universidade Federal de. Alfenas - UNIFAL-MG. eduardosalgado@unifal-mg.edu.br ${ }^{2}$ Professor adjunto do Instituto de Ciências Exatas, Professor permanente do Programa de Pós-Graduação em Ciências Ambientais - PPGCA - UNIFAL-MG. eduardosalgado@unifal-mg.edu.br

${ }^{3}$ Bacharel em Biotecnologia, Mestranda no PPGCA - UNIFAL-MG. uzanaeda@hotmail.com
\end{abstract}

Recebido em: 06/11/2015 - Aprovado em: 19/07/2016 - Disponibilizado em: 18/12/2016

\begin{abstract}
RESUMO
Com a globalização, a internacionalização do mercado e o consequente aumento da competitividade entre empresas, tem crescido a preocupação entre gestores a respeito da importância de fatores como produtividade e a qualidade dos seusprodutos. Parasatisfazer as expectativas do cliente e superar a qualidade da produção das empresas concorrentes, as organizações têm empregadovariadas estratégias de gestão. O objetivo do presente trabalho foi estudar o uso de indicadores de processos, ferramentas da qualidade e a da comunicação entre funcionários no âmbito organizacional. Um estudo de caso foi realizado em uma unidade de uma empresa de biotecnologia localizada em Petrolina-PE, Brasil. Realizou-se a aplicação de questionário para sete funcionários da empresa,com questões relativas aos temas de interesse mencionados. Os resultados mostraram que a empresa possui missão e valores definidos e há consciência e interesse entre os gestores na implantação de estratégias para melhoria da qualidade. No entanto, problemas na comunicação e desconhecimento a respeito das ferramentas da qualidade podem limitar o crescimento da empresa no setor de biotecnologia.
\end{abstract}

Palavras-chave:Comunicação.Ferramentas de gestão. Indicadores de performance. Empresas de biotecnologia. Melhoria da qualidade.

\begin{abstract}
With the globalization, the internationalization of the market and the resulting increase in competitiveness between companies has grown the concern among managers about the importance of productivity and quality of their products. To meet the customer expectations and surpass the production quality of competing companies, organizations have employed various management strategies. The objective of this work was to study the use of process indicators, quality tools and communication between employees at the organizational level. A case study was carried out in a unit of a biotechnology company located in Petrolina, Pernambuco, Brazil. It was held on a questionnaire for seven employees, with questions about the topics of interest mentioned. The results showed that the company has defined mission and values and there is awareness and interest among managers in the implementation of strategies for improving quality. However, problems in communication and unfamiliarity with the quality tools may limit the company's growth in the biotechnology sector.
\end{abstract}

Keywords: Communication. Management tools. Performance indicators. Biotechnology companies. Quality improvement.

\section{INTRODUÇÃO}

A globalização tem proporcionado a oportunidade para empresas expandirem seus mercados de negócios e disponibilizarem seus produtos e serviços nas mais diversas regiões do mundo. A ampliação do comércio internacional, que vem contribuindo para o crescimento de muitas empresas, também tem tornado o mercado cada vez mais competitivo, forçando empresas a buscarem alternativas para se destacar no mercado 
(ENSSLIN; ENSSLIN, 2010) e reduzir o impacto da diversidade de práticas nacionais. Somado ao surgimento de consumidores cada vez mais exigentes, esses fatores vem impulsionado empresas a aplicarem práticas da melhoria na gestão estratégica, tornando-as mais preparadas frente às alterações dos contextos sociais e econômicos (GONZALEZ; MARTINS, 2007; DA SILVA, LIMA, 2015).

O lançamento de novos produtos, soluções e serviços exige que a qualidade destes esteja dentro dos padrões exigidos (ALMEIDA;MIGUEL; DE CARVALHO, 2011; MATTAR, 1982), principalmente em áreas em constante inovação como a biotecnologia. Assim, torna-se indispensável à existência de sistemas de gestão e ferramentas que possibilitem uma melhoria de forma contínua, sem causar grandes impactos na empresa, aumentando a demanda de seus produtos e colocando a empresa em posição de destaque para os consumidores (MARTINS, 1998).

A clareza na transmissão das informações interna entre funcionários e lideranças também é uma importante estratégia para melhoria nos processos e também no ambiente de trabalho. Para atingir o sucesso na implantação de estratégias para melhoria na gestão, é necessário que todos os funcionários afetados pelas mudanças estejam conscientes sobre a importância dessas alterações para a qualidade da produção.
O monitoramento das atividades desenvolvidas na organização, através de medições do desempenho também pode auxiliar nesse processo de melhoria contínua da qualidade dentro da empresa, permitindo verificar como está o desenvolvimento da empresa, quais as prováveis razões que determinam a situação atual e quais ações podem ser tomadas visando melhorias (ATTADIA; MARTINS, 2003). Neste contexto, estabelecer um sistema de medição do desempenho baseada em indicadores mostra-se fundamental para o estabelecimento de metas de melhorias no processo.

O objetivo desse trabalho foi avaliar a importância de indicadores de desempenho, ferramentas da qualidade e, de forma complementar, da avaliação do sistema de comunicação, em uma nova unidade de pesquisa de uma grande multinacional do setor de biotecnologia. A importância da pesquisa justifica-se pelo fato de que não foram encontrados trabalhos na literatura referentes aos tópicos abordados especificamente para empresas de biotecnologia.

\section{REVISÃO DA LITERATURA}

\subsection{EMPRESAS DE BIOTECNOLOGIA}

O setor de biotecnologia representa uma das indústrias mais importantes em economias que visam desenvolver uma sólida 
base em Pesquisa e Desenvolvimento (P\&D) (KANG; PARK, 2012). Dentro dessas empresas, os conhecimentos dessa área são aplicados por meio de variadas técnicas de base biológica com caráter tecnológico, visando melhorias na prestação de serviços e obtenção de produtos (SAMAAN et al., 2012).

Atualmente, a biotecnologia é considerada uma das áreas do conhecimento prioritárias no Brasil, atraindo a atenção da indústria e de instituições de pesquisa e ensino, principalmente do setor público (MATIAS; VIEIRA; FONTENELE, 2014). Principalmente nos últimos trinta anos, seu crescimento foi bastante favorecido no país devido à necessidade de ampliação da base da pesquisa, com a criação de diversos programas de apoio governamentais, visando o desenvolvimento e estruturação da ciência e biotecnologia no Brasil (BIANCHI, 2013; SIMÕES, MARTINS, 2013).

Um dos pontos principais dessas políticas públicas de incentivo, dentre outros, tem sido a criação de novos empreendimentos de base biotecnológica (BIANCHI, 2013). Essas indústrias tem apresentado importante participação em setores econômicos tradicionais como saúde, alimentos, agricultura e manejo do meio ambiente, promovendo progressos no tratamento de doenças e descoberta de novos medicamentos, manipulação e reprodução animal e vegetal, pesquisas e melhorias na área de alimentos, promoção do uso sustentável dos recursos naturais e recuperação de áreas contaminadas, entre outras áreas de grande importância social (DA SILVA; BAYDOUN; BADRAN, 2002).

$\mathrm{Na}$ área agrícola, a inserção da biotecnologia apresenta fundamental importância, em especial quando relacionada à agricultura sustentável e conservação dos recursos naturais, através da redução da aplicação de compostos tóxicos, aumento da produtividade agrícola e melhoria na qualidade dos alimentos produzidos (PESSÔA et al. 2006; OLIVEIRA et al., 2012).

\subsection{INDICADORES DE DESEMPENHO}

A medição do desempenho, um importante elemento do gerenciamento empresarial, vem sendo muito aplicada devido a necessidade das empresas em buscarem métodos rápidos e eficazes para a tomada de decisões estratégicas, em um meio com alta competitividade como o ambiente de negócios. Nesse contexto, indicadores de desempenho são utilizados para pelas empresas para monitorar as estratégias relacionadas ao seu comportamento atual e futuro (FERREIRA et al., 2008).

Para a compreensão do processo de medição de desempenho de um processo, o conhecimento a respeito do conceito de desempenho pode ser aplicado (MARTINS, 
COSTA NETO, 2008). Segundo Lebas (1995), desempenho pode ser definido como:

“... gestão e implantação dos componentes do modelo causal que levam à realização dos objetivos afirmados, dentro das restrições específicas para a empresa e para a situação..." (LEBAS, 1995).

Os indicadores de desempenho são parâmetros utilizados para medir a eficácia e eficiência no processo. Estes indicadores anteriormente eram basicamente relacionados a fatores financeiros, mas atualmente é há uma ampla gama de indicadores relacionadosàs estratégias e as operações envolvidas no processo (SAMPAIO;

SARAIVA; DOMINGUES, 2012).

A utilização dos indicadores de desempenho possui importância fundamental para empresas que se preocupam com seu posicionamento no mercado, pois a qualidade da produção só pode ser assegurada quando há grande rastreabilidade e controle nos processos que resultam nesse produto ou serviço final prestado (SOARES, CARVALHO, 2007).

Esse processo de medição também torna mais fácil o estabelecimento de políticas de qualidade (PINTO; GOMES, 2010). A qualidade é o que determina se o produto atende às requisições do cliente, se o tempo de produção é aperfeiçoado, o custo é ideal, e os valores da empresa são devidamente atendidos.

\subsection{PROGRAMAS E FERRAMENTAS DA} QUALIDADE

Segundo Barbêdo (2004), antes de 1900, os parâmetros de qualidade ficavam à determinação dos bons artesãos e operadores. Somente a partir desse período ocorreu o inicio da supervisão dos processos, seguido pela inspeção, controle estatístico da qualidade, a motivação do uso de qualidade e por fim a implantação de sistemas de gestão de qualidade. Quase um século foi então necessário para que os Sistemas de Gestão de Qualidade (SGQ) fossem criados e implantados, acompanhando diversas mudanças no foco da qualidade, que já foi centrado no processo, no produto, no cliente, na empresa, originando a busca atual pela qualidade total.

Para o estabelecimento de práticas de melhoria contínua, a obtenção da certificação ISO 9001, que apresenta os requerimentos para a implantação de um SGQ em conformidade com a norma é importante (ALBUQUERQUE; BRONNENBERG; CORBETT, 2007). O sucesso desta implantação é dependente, no entanto, da finalidade com que a certificação é adotada, ou seja, se a empresa visa realizar melhorias ou se simplesmente está certificando suas estruturas por pressão externa de consumidores

(PSOMAS;

KAFETZOPOULOS; FOTOPOULOS,2010).

É importante notar que os gestores que decidem adotar a norma da qualidade por 
pressão de mercado, muitas vezes não conhecem adequadamente as características dessa certificação.

Para a implantação de sistemas da qualidade nas organizações podem ser utilizados indicadores de desempenho aliados às ferramentas de qualidade. Há sete ferramentas básicas de qualidade descritas por Vasconcelos e Pereira (2011):

"Estratificação: Consiste no agrupamento da informação (dados) sob vários pontos de vista, de modo a focalizar a ação [...]

Folha de Verificação: Formulário no qual os itens a serem verificados para observação do problema já estão impressos, com o objetivo de facilitar a coleta e o registro dos dados.

Gráfico de Pareto: Um problema pode ser atribuído a um pequeno número de causas. Representado por um gráfico de barras verticais que dispõe a informação de forma a tornar evidente e visual a priorização de temas.[...]

Diagrama de Causa e Efeito: Apresenta a relação existente entre um resultado de um processo (efeito) e os fatores (causas) do processo [...].

Histograma: Gráfico de barra que dispõe as informações de modo que seja possível a visualização da forma da distribuição de um conjunto de dados, e também a percepção da localização do valor central.

Diagrama de Dispersão: Gráfico utilizado para a visualização do tipo de relacionamento existente entre duas variáveis. Estas variáveis podem ser duas causas de um processo, uma causa e um efeito do processo ou dois efeitos do processo.

Gráfico (carta) de Controle: Utilizado para detectar causas especiais e causas comuns $[\ldots] "$.

Outro grupo de ferramentas da qualidade amplamente abordado na literatura é composto por ferramentas como o 6sigma,
5W 2H, PDCA, 5S, Questionários, Diagrama de Pert, Gráficos e Brainstorming.

\subsection{COMUNICAÇÃO}

A comunicação é peça fundamental no bom desenvolvimento de uma companhia, falhas de comunicação podem trazer atrasos e falhas no processo (ANDRADE; RIETOW; MORAES, 2012).

\subsection{A EMPRESA DE BIOTECNOLOGIA EM PETROLINA - PE}

A empresa de biotecnologia em estudo foi inaugurada oficialmente em março de 2013, na cidade de Petrolina - PE. Ela é vista como uma peça fundamental no desenvolvimento da multinacional no Brasil, sendo desenvolvidas nessa unidade atividades de suporte a outros times.

Petrolina é uma cidade no sertão nordestino, localizada às margens do rio São Francisco. Muito conhecida pela produção e exportação de frutas de clima tropical, a cidade possui canais que permitem o desenvolvimento de sistemas de irrigação. Com clima bem definido sem muitas chuvas, a região do vale do São Francisco é bastante procurada por produtores que desejam ter suas plantações em regiões nas quais é possível prever o clima e não depender totalmente de fatores climáticos para ter boa produtividade. 
Essa empresa é mundialmente conhecida pelo uso da biotecnologia, produtora de diversos produtos de base biotecnológica, sendo o principal o milho, seguido pela soja, algodão, cana, sorgo, hortaliças e outros produtos de menor impacto no mercado. Uma importante característica dessa empresa é o fato de ser amplamente criticada pela forma inovadora com conduz sua produção, sendo essas críticas muitas vezes relacionadas ao emprego de técnicas relacionadas à própria biotecnologia.

\section{METODOLOGIA}

\subsection{OBTENÇÃO DOS DADOS}

Um estudo de caso sobre a utilização de indicadores de desempenho, a comunicação e as ferramentas da qualidade foi realizado na unidade da empresa de biotecnologia em Petrolina, com o intuito de avaliar e trazer possíveis melhorias.

O estudo de caso é uma forma de avaliação, realizada através da comparação entre as práticas adotadas e as recomendadas pela literatura. Para a avaliação da situação na empresa, foi utilizada uma pesquisa de levantamento. Ela consiste em uma prática que visa obter dados relativos aos conhecimentos e opiniões sobre determinado assunto diretamente com um grupo de pessoas de interesse, por meio de questionário ou entrevistas pessoais, para obter informações mais objetivas e precisas (SAMAAN et al., 2012).

No presente estudo, sete funcionários correspondentes a três diferentes equipes dentro da unidade da Empresa de biotecnologia em Petrolina, foram questionados sobre:

1.Importância dos indicadores de desempenho dentro das equipes de trabalho;

2. Ferramentas da qualidade utilizadas;

3. Gestão da comunicação.

No quadro 1 a seguir encontram-se as características individuais de cada funcionário.

Quadro 1 - Características dos especialistas avaliados

\begin{tabular}{|c|c|c|}
\hline Característica & Função & $\begin{array}{c}\text { Formação } \\
\text { Acadêmica }\end{array}$ \\
\hline Entrevistado A & Líder de grupo & $\begin{array}{c}\text { Graduação em } \\
\text { Agronomia. } \\
\text { Mestrado e } \\
\text { Doutorado em } \\
\text { áreas correlatas. }\end{array}$ \\
\hline Entrevistado B & Líder de grupo & $\begin{array}{c}\text { Graduação em } \\
\text { Agronomia. } \\
\text { Mestrado e } \\
\text { Doutorado em } \\
\text { áreas correlatas. }\end{array}$ \\
\hline Entrevistado C & $\begin{array}{c}\text { Líder de grupo } \\
\text { Eraduação em } \\
\text { Agronomia. } \\
\text { Mestrado e } \\
\text { Doutorado em }\end{array}$ \\
\hline Éreas correlatas.
\end{tabular}

A pesquisa foi baseada em um trabalho feito com pequenas e medias 
empresas em Portugal sobre a importância de indicadores de desempenho e aplicação de ferramentas da qualidade em empresas portuguesas (SOUSA et al., 2005). O questionário do artigo foi adequado às situações do pólo de pesquisa da empresa de biotecnologia em Petrolina e as áreas presentes na unidade.

O questionário foi traduzido e adaptado para que todas as questões tivessem aplicabilidade.

\subsection{AVALIAÇÃO DOS RESULTADOS}

Com as informações obtidas nas entrevistas com os profissionais, foi realizada uma comparação entre as respostas, visando realizar uma avaliação geral da situação atual com relação aos tópicos abordados, do ponto de vista dos funcionários da unidade. Esse procedimento teve os seguintes objetivos: avaliar os pontos de melhoria existentes, para aperfeiçoar os processos através da implantação de indicadores de desempenho, melhorar o sistema de comunicação e avaliar o conhecimento das pessoas sobre as ferramentas da qualidade.

\section{RESULTADOS E DISCUSSÃO}

\subsection{INDICADORES DE DESEMPENHO}

O primeiro questionamento estava relacionado à missão e os valores da empresa.
Segundo Al-Darrab, Gulzar e Ali (2013), para a implantação de Sistemas de Qualidade, é preciso que a empresa tenha uma cultura bem definida, que se inicia com o estabelecimento e difusão da missão, visão e valores da empresa, e prossegue com a preocupação com o meio ambiente, representada por processos produtivos limpos.

Em relação à empresa em estudo, a preocupação com a sustentabilidade é uma forte característica da cultura dessa empresa de biotecnologia. Os resultados também demonstraram que a empresa se preocupa em mostrar a todos os seus funcionários, dos mais diversos setores, qual é a sua missão e quais são os seus valores. A difusão das informações entre os funcionários promove um alinhamento entre todos os membros da empresa com relação aos objetivos do seu trabalho. Esta característica apresenta especial importância para empresas do setor de biotecnologia, que estão particularmente sujeitas a um excesso de ideias e informações que pode desestruturar a empresa, devido ao rápido desenvolvimento de novas tecnologias e à necessidade de constantes reciclagens, além das inúmeras possibilidades de novas oportunidades de negócios.

Também em relação à importância da difusão da missão e valores entre os diversos níveis hierárquicos da empresa, é preciso avaliar o que cada grupo tem feito com essa informação. Esta avaliação pode ser feita de diversas formas, sendo uma delas a utilização 
de indicadores de desempenho que mostrem a eficiência de cada atividade, produtividade, erros, gastos, entre outros. A partir destes indicadores, é possível verificar se as atividades desenvolvidas estão alinhadas com as necessidades da companhia, ou seja, se há uma preocupação efetiva entre os funcionários (GARENGO; BIAZZO, 2013).

A avaliação realizada pelos entrevistados mostrou que os três times conhecem a importância dos indicadores de desempenho e como estes podem ser benéficos aos processos realizados. Contudo, dentre os três, somente dois times frequentemente faziam a utilização destes indicadores, empregando planilhas de verificação para avaliação periódica de seus processos.

O grupo que não utilizava planilhas, por sua vez, não controlava minuciosamente os seus processos, sendo esse controle realizado de forma superficial e sem documentos. A produtividade no campo era priorizada, no entanto ela não possui controle efetivo.Durante a realização deste trabalho muitas mudanças ocorreram no grupo e os processos passaram a ter maior controle e documentação efetiva.

Um mês após a aplicação do questionário, o grupo que relatou não fazer uso dos indicadores de qualidade recebeu um novo líder, que estabeleceu muitos parâmetros de qualidade, e teve como primeira atitude requerer dos funcionários alguns a documentação relativa aos indicadores de desempenho em cada uma das atividades.

Kammoun e Aouni (2013) mostraram em seu trabalho a importância do comprometimento da liderança com a obtenção de resultados, pois a ausência desse compromisso poderia impedir o progresso da companhia. É fundamental que a liderança tenha um posicionamento forte e definido quanto à importância e utilização dos parâmetros, demonstrando aos funcionários que os indicadores de desempenho trazem a necessidade de documentação contínua. Esse esforço pode trazer uma melhor visão do que tem ocorrido no setor e seus processos e ainda trazermelhorias e outras necessidades de aperfeiçoamento. $\mathrm{Na}$ unidade, foi constatado que a liderança é bastante comprometida com o trabalho e determinada a atingir os objetivos da companhia.

Para o estabelecimento de indicadores de desempenho é preciso haver um alinhamento entre as boas práticas e os objetivos da empresa. É necessário conhecer o sistema de gestão e, mais especificamente, a gestão dos processos desenvolvidos. Nesse contexto, segundo Moraes (2012), é preciso que os indicadores de produtividade tenham alinhamento com os ideais da companhia, há a necessidade do estudo dos processos utilizados e dentro destes processos e é fundamental avaliar aqueles que são mensuráveis e aqueles que podem trazer dados importantes. Baseado nessas 
afirmações realizou-se o questionamento a respeito de quais indicadores seriam os mais importantes com relação aos ideais da empresa.

$\mathrm{O}$ indicador de desempenho considerado essencial pelos funcionários da empresa foi a produtividade, que englobaria em todo o processo,desde o trabalho no campo até a entrega do produto ao cliente. Para estabelecer este indicador, é preciso determinar os parâmetros importantes para o processo como o tempo gasto para o processo e para cada uma das etapas, a quantidade de material utilizado, a quantidade de produto entregue, o número de trabalhadores necessários. Determinados estes parâmetros, é possível definir e calcular indicadores que possibilitem melhor controle do processo.

O parâmetro relativo ao tempo gasto para entrega do produto aos clientes pode avaliar o quanto o serviço de envio é eficiente, o que consequentemente afetará de forma direta a satisfação dos clientes. É fundamental que a logística da empresa cumpra o prazo requerido pelo cliente, especialmente com relação a produtos biotecnológicos e de pesquisa. A eficiência no envio torna o fluxo do trabalho contínuo e constante, além de possibilitar o cumprimento dos cronogramas.

A qualidade é outro parâmetro avaliado como fundamental para o processo. Processos de qualidade devem ser executados de modo apurado, eficaz e eficiente. A qualidade desses processos constitui uma categoria de indicadores que deve ser avaliada por diversos níveis de stakeholders, uma vez que é preciso conhecer as expectativas dos stakeholders para estabelecer o nível de qualidade desses processos (GOTZAMANI, 2013).

Entre as vantagens que um processo de qualidade oferece para a empresa estão a redução de gastos excessivos, controle sobre o excesso de mão de obra, a possibilidade de maior qualidade dos seus produtos, e principalmente, uma melhoria na eficiência do processo como um todo.

Entretanto, é necessário constantemente reavaliar esses processos e atualizar esses indicadores de desempenho, para que eles estejam adequados à situação encontrada na empresa, visando minimizar o esforço excessivo para identificar indicadores inadequados a situação e que não trazem respostas conclusivas (MARTINS, 2008). Para isso é preciso que haja uma boa aceitação na introdução de novos indicadores, e que todos percebam sua aplicabilidade dentro da empresa (SALMADOR; BUENO; MARANHAO, 2013).

Empresas que possuem como valores iniciativa e visão deverão se desenvolver e trazer novidades aos seus processos, com o objetivo de torná-las mais dinâmicas. Os entrevistados relataram, no entanto, a presença de algumas barreiras que impossibilitariam esta renovação na empresa, 
tais como a falta de treinamento dos funcionários, que seria uma dificuldade tanto para a renovação dos processos como para a obtenção de dados para indicadores de desempenho.

Para renovar os processos, é necessário primeiramente treinar o grupo de funcionários. No entanto, dentro de uma cultura habituada aos processos constantemente executados, há certa dificuldade em inserir novas metodologias e formas diferentes de atuação. Esta complicação muitas vezes está ligada ao próprio sistema de liderança, em que os próprios líderes não estão dispostos a aceitar mudanças e aprender coisas novas. Essa barreira ligada ao sistema de liderança pode ainda estar relacionada à falta de comunicação, terceiro ponto visto como crítico na instauração de novos indicadores e renovação dos processos, e que constitui um problema muito recorrente dentro de empresas.

A comunicação dentro da empresa precisa ser objetiva e eficiente para que as novidades apresentadas aos processos sejam bem vistas e a necessidade de mudanças seja clara a todos aqueles afetados por estas atualizações. Em vista da importância desse tópico, optou-se por estender a pesquisa além da análise dos indicadores de desempenho e ferramentas da qualidade. Logo, uma ramificação do trabalho foi feita, tendo em vista as constantes reclamações atribuídas à comunicação.

\subsection{COMUNICAÇÃO}

Nesse tópico da pesquisa, foram realizados questionamentos para avaliar as condições da gestão de informação atualmente encontradas na unidade, a necessidade de documentação dessas informações e a importância da clareza no processo de comunicação. Dentre as questões abordadas, a única respondida de forma unanime por todos os entrevistados foi a relacionada ao acesso que os funcionários têm aos meios de comunicação.

Os entrevistados afirmaram que todos os funcionários possuíam livre acesso aos meios de comunicação na empresa. Contudo, quando questionados sobre a satisfação referente aos meios de comunicação disponíveis, os entrevistados demostraram que há pontos que precisariam ser melhorados. Apesar da disponibilidade de computadores com acesso à Internet e de quadros de avisos, a empresa recomendava a documentação de qualquer informação e solicitação, preferencialmente via e-mail, para que todos os processos realizados fossem documentados. Entretanto, os gestores da empresa de biotecnologia sinalizaram que nem sempre as informações eram documentadas, uma falha relacionada tanto à indivíduo que envia a informação, que não vê 
a importância ou necessidade de documentar a informação, como ao indivíduo que recebe a informação, e na maioria das vezes não requer a comunicação na forma de documento.

Com relação à gestão da informação, Andrade,Rietow e Moraes, (2012) demonstraram a importância de estabelecer um padrão de comunicação, pois, esta comunicação, quando feita de forma eficaz e sem "ruídos", permite que a informação seja transmitida de forma mais clara e entregue com maior eficiência aos mais diversos níveis da organização. Na empresa em estudo, porém, verificou-se que não há um padrão na escolha do meio de comunicação a ser utilizado e a importância da informação a ser transmitida, sendo este fato identificado como uma das principais falhas na transmissão de informações na empresa.

A padronização de processos relativos ao fluxo de informação traz uma série de benefícios à empresa: melhor compreensão sobe as atividades realizadas, as expectativas de resultados se tornam mais claras, e as vantagens se estendem a melhorias na convivência dentro do ambiente de trabalho, pois uma maior clareza na transmissão da informação leva a um melhor entendimento entre os funcionários. Entre os resultados das entrevistas da pesquisa, a clareza e a objetividade da informação foram os pontos de melhoria observados.

\subsection{PROGRAMAS E FERRAMENTAS DA QUALIDADE}

$\mathrm{Na}$ última etapa, foi avaliado o conhecimento que os líderes e funcionários apresentavam sobre a certificação ISO 9001, que orienta a implantação de Sistemas de Gestão da Qualidade e a respeito das ferramentas mais frequentemente utilizadas na área da qualidade.

Com relação à certificação ISO 9001, os questionários abordavam o conhecimento que os líderes tinham sobre a norma e suas implicações no setor (LLACH; BERNADO; MARIMON, 2011). Até o momento do estudo, a unidade não possuía a certificação, mas os entrevistados reconheceram a importância da norma, demonstraram conhecer a certificação e identificar seu objetivo de orientar a implantação de SGQs nos processos e produtos. Além disso, os funcionários confirmaram saber a importância que uma futura certificação ISO 9001 representaria para a unidade, por conferir à empresa certificada melhorias na sua imagem e por demonstrar a preocupação dessa organização com a qualidade em seus negócios, uma característica bem vista pelos clientes.

Para utilizar as ferramentas da qualidade na busca pela melhoria contínua na empresa, é preciso conhecê-las, e tê-las alinhadas com os processos realizados dentro da organização (HERAS-SAIZARBITORIA; 
CASADESÚS; MARIMÓN, 2013). Além da ISO 9001, os gestores também foram questionados a respeito de algumas ferramentas da qualidade que, conforme Rusjan e Alic (2010), possibilitam detectar, prevenir e corrigir falhas na empresa.

$\mathrm{Na}$ empresa de biotecnologia, os entrevistados demonstraram baixo conhecimento a respeito dos dois grupos de ferramentas dos quais foram questionados, o que mostra a necessidade de maior capacitação dos líderes sobre as ferramentas da qualidade. Autores como Kammoun e Aouni (2013) comentam que a falta de conhecimento e comprometimento dos líderes, pode limitar o desenvolvimento dos processos produtivos e, no caso dessa empresa em Petrolina, embora os gestores estejam constantemente buscando soluções para as melhorias dos seus processos, o pouco conhecimento sobre as ferramentas da qualidade disponíveis para promover tais melhorias poderia limitar a evolução dessa empresa dentro do setor de biotecnologia.

\section{CONSIDERAÇÕES FINAIS}

$\mathrm{Na}$ empresa de biotecnologia em estudo, a missão e valores são bem definidos e divulgados aos stakeholders, o que possibilita direcionar as atividades que irão culminar no produto. Os líderes e gerentes na unidade de Petrolina parecem estar interessados na instauração de boas práticas, fundamentação dos processos e busca pela qualidade, apesar de ainda desconhecerem algumas ferramentas que podem ser aplicadas no processo de melhoria.

Atualmente, na unidade da empresa estudada, os indicadores de desempenho tem sua importância reconhecida pelos entrevistados, mas eles ainda não são utilizados, o que seria um primeiro ponto de melhoria na organização.

Há consenso entre os gestores entrevistados sobre a necessidade da adoção da certificação ISO 9001. No entanto, para obter as melhorias desejadas na qualidade é importante que as pessoas responsáveis por identificar os pontos de melhoria possuam o conhecimento necessário para tal e reconheçam a importância da norma para a melhoria da gestão.

Com relação à comunicação percebeuse a necessidade do estabelecimento de um padrão do fluxo de informação. Além disso, as barreiras na comunicação podem vir a causar eventuais problemas nos processos. A unidade de Petrolina está em constante crescimento e necessita que seus processos ocorram de forma contínua e eficiente. A comunicação clara e objetiva e o fluxo de informação bem estabelecido podem ser decisivos na melhoria da eficiência do processo.

Por fim, em relação à avaliação do conhecimento entre os funcionários a respeito das ferramentas da qualidade, os resultados 
dos questionários indicaram que há pouco conhecimento entre eles sobre essas ferramentas, que são de grande valor na gestão dos processos. Para implantação de práticas da qualidade, principalmente em um setor em que ela é tão exigida como a biotecnologia, é importante que haja uma maior concentração de informações sobre estas ferramentas e sua utilização dentro dessa empresa.

\section{REFERÊNCIAS BIBLIOGRÁFICAS}

ALBUQUERQUE, P.; BRONNENBERG, B. J.; CORBETT, C. J. A spatiotemporal analysis of the global diffusion of ISO 9000 and ISO 14000 certification. Management Science, n. 53, v.3, p. 451-468, 2007.

AL-DARRAB, I. A.; GULZAR, W. A.; ALI, K. S. Status of implementation of safety, quality and environmental management systems in Saudi Arabian industries. Total Quality Management \& Business Excellence, n.24, v. 3-4, p. 336-354, 2013.

ALMEIDA, L.; MIGUEL, P.C.; DE CARVALHO, M. M. Aplicação do gerenciamento de projetos no processo de desenvolvimento de novos produtos - um caso exploratório. Revista de Gestão e Projetos, n.2, v. 2, 2011.

ANDRADE, M. J. O. DE; RIETOW, V.; MORAES, P. E. S. O importante processo da comunicação para a gestão da qualidade e a implementação das normatizações ISO 9001.

Revista Intersaberes, n.2, v.3, p.133-148, 2012.

ATTADIA, L. C. L.; MARTINS, R. A. Medição de desempenho como base para evolução da melhoria contínua. Revista Produção, n.13, v.2, p. 33, 2003.
BARBÊDO, S. A. D. 2004. Sistema de gestão da qualidade em serviços: estudo de caso em uma biblioteca universitária. Tese (Doutorado em Engenharia de Produção) Universidade Federal de Itajubá - UNIFEI, 134 p, 2004.

BIANCHI, C.A Indústria Brasileira de Biotecnologia: montando o quebra-cabeça. RevistaEconomia\&Tecnologia, n.9, v.2, 2013.

DA SILVA, E. J.; BAYDOUN, E.; BADRAN, A. Biotechnology and the developing world.Electronic Journal of Biotechnology,n.5, v.1, p. 1-2, 2002.

DA SILVA, E. H. D. R.; LIMA, E. P. O estudo de indicadores de desempenho sob o enfoque da gestão estratégica organizacional. Revista GEPROS, n.10, v.3, p. 159, 2015

ENSSLIN, L.; ENSSLIN, S. R. Modelo para avaliar o desempenho de operadores logísticos - um estudo de caso na indústria têxtil. Gestão \& Produção, n.17, v.4, 693$705,2010$.

FERREIRA, M. P. et al.Gestão por indicadores de desempenho: resultados na incubadora empresarial tecnológica.Produção, n.18, v.2, p.302-318, 2008.

GARENGO, P.; BIAZZO, S. From ISO quality standards to an integrated management system : an implementation process in SME. Total Quality Management \& Business Excellence, n.24, v.3-14, p. 310335, 2013);

GONZALEZ, R. V. D.; MARTINS, M. F.Melhoria contínua no ambiente ISO 9001: 2000: estudo de caso em duas empresas do setor automobilístico. Revista Produção, n.17, v.3, p.592-603, 2007.

GOTZAMANI, K. Results of an empirical investigation on the anticipated improvement areas of the ISO $9001: 2000$ standard. Total 
Quality Management \& Business

Excellence, n.21, v.6, p. 687-704, 2010.

HERAS-SAIZARBITORIA, I.; CASADESÚS, M.; MARIMÓN, F. The impact of ISO 9001 standard and the EFQM model : The view of the assessors. Total Quality Management \& Business

Excellence, n.22, v.2, p.197-218, 2013.

KAMMOUN, R.; AOUNI, B. ISO 9000 adoption in Tunisia : experiences of certified companies. Total Quality Management \& Business Excellence, n.24, p.259-274, 2013.

KANG, K-N; PARK, H. Influence of government R\&D support and inter-firm collaborations on innovation in Korean biotechnology SMEs.Technovation, n.32, v.1, p.68-78, 2012.

LEBAS, M. J. Performance measurement and performance management. International Journal of Production Economics, n.41, v.1, p.23-35, 1995.

LLACH, J.; BERNARDO, M.; MARIMON, F. ISO 9001 diffusion analysis according to activity sectors. Industrial Management \& Data Systems, n.111, v.2, p. 298-316, 2011.

MARTINS, R. A. Efeitos da adoção do modelo do Prêmio Nacional da Qualidade na medição de desempenho : estudos de caso em empresas ganhadoras do prêmio. Gestão \& Produção, n.15, v.2, p.247-259, 2008.

MARTINS, R. A. Indicadores de desempenho para a gestão pela qualidade total: uma proposta de sistematização. Gestão \& Produção, v. 5, n. 3, p. 298-311, 1998.

MARTINS, R. A.; COSTA NETO, P. L.de O. Indicadores de desempenho para a gestão pela qualidade total: uma proposta de sistematização. Gestão \& Produção, n.5, v.3, p.298-311, 1998.

MATIAS, F.; VIEIRA, P. I. L.; FONTENELE, H. A. Avaliação do perfil de investimentos em biotecnologia no Brasil.
Cadernos de Prospecção, n.7, v.3, p.314, 2014.

MATTAR, F. A. Informação de marketing e o sucesso no lançamento de novos produtos.

Revista de Administração, n.17, v.4, p. 2344, 1982.

MORAES, L. 2012.Metodologia para auxiliar na definição de indicadores de desempenho para a gestão da tecnologia médico-hospitalar. Tese (Doutorado em engenharia Elétrica), Universidade Federal de Santa Catarina - UFSC, 242 p, 2012.

OLIVEIRA, A. M. X; et al. A biotecnologia aplicada ao melhoramento genético vegetal: controvérsias e discussões. Revista da Universidade Vale do Rio Verde,n.10, v.1, p.339-361, 2012.

PESSÔA, L. T.; CARVALHO, D.; PEREIRA, N. Transgênicos e indicadores ambientais. Engenharia Ambiental, n.3, v.2, p.86-106, 2006.

PINTO, L.; GOMES, J. Custos da qualidade: um estudo de caso na panificadora Golden vital. Revista de Informação Contábil, n.4, v.27, p.57-76, 2010.

PSOMAS, E. L.; Kafetzopoulos, D. P.;

Fotopoulos, C. V. Critical factors for effective implementation of ISO 9001 in SME service companies. Managing Service Quality, n.20, v.5, p.440-457, 2010.

RUSJAN, B.; ALIC, M. Capitalizing on ISO 9001 benefits for strategic results.

International Journal of Quality \&

Reliability Management, n.27, v.7, 756-778, 2010.

SALMADOR, P. M.; BUENO, E.; MARANHAO, R. Total quality management: A critical analysis from a complexity approach. Total Quality Management, n.19, v.5, p.513-533, 2008.

SAMAAN, M. et al. Identificação dos fatores críticos de sucesso no desenvolvimento de 
produtos de empresas de biotecnologia do estado de Minas Gerais. Produção, n.22, v.3, p.436-447, 2012.

SAMPAIO, P.; SARAIVA, P.;

DOMINGUES, P. Management systems: integration or addition?International Journal of Quality \& Reliability

Management, n.29, v.4, p. 402-424, 2012.

SIMÕES, R.; MARTINS, A. Inovação e biotecnologia: atributos urbanos e estrutura científica. In: XLI Encontro Nacional de Economia, 2014. Anais... Belo Horizonte, UFMG, 2014.

SOARES, S.; CARVALHO, H. A.

Implementação de indicadores de qualidade e desempenho através do gerenciamento por projeto. Estudo de caso dos locais de produção de uma empresa em Curitiba. 2007.

SOUSA, S. D. et al. Performance measures and quality tools in Portuguese small and medium enterprises: survey results". Total Quality Management \& Business Excellence, n.16, v.2, p.277-307, 2005.

VASCONCELOS, N. V. C.; PEREIRA, C. B. Análise do processo logístico através das ferramentas da qualidade: um estudo de caso na DDEX-direct to door express. INGEPROInovação, Gestão e Produção, n.3, v.2, p.059-071, 2011. 